\title{
Further Development of Feedback Control of Cavity Flow Using Experimental Based Reduced Order Model
}

\author{
E. Caraballo*, X. Yuan ${ }^{\dagger}$, J. Little ${ }^{\ddagger}$, M. Debiasi ${ }^{\S}$, A. Serrani** ${ }^{*}$, J. H. Myatt ${ }^{\dagger \dagger}$, and M. Samimy ${ }^{\ddagger}$ \\ Gas Dynamics and Turbulent Laboratory; Collaborative Center for Control Science \\ The Ohio State University, Columbus, Ohio
}

\begin{abstract}
In our recent work we presented preliminary results for subsonic cavity flow control using a reduced-order model based feedback control derived from experimental measurements. The model was developed using the Proper Orthogonal Decomposition of PIV images in conjunction with the Galerkin projection of the Navier-Stokes equations onto the resulting spatial eigenfunctions. A linear-quadratic optimal controller was designed to reduce cavity flow resonance by controlling the time coefficient and tested in the experiments. The stochastic estimation method was used for real-time estimation of the corresponding time coefficients from 4 dynamic surface pressure measurements. The results obtained showed that the controller was capable of reducing the cavity flow resonance at the design Mach 0.3 flow, as well as at other flows with slightly different Mach number. In the present work we present several improvements made to the method. The reduced order model was derived from a larger set of PIV measurements and we used 6 sensors for the stochastic estimation of the instantaneous time coefficients. The reduced order model so obtained shows a better convergence of the time coefficients. This combined with the 6sensor estimation improves the control performance while using a scaling factor closer to the theoretically expected value. The controller also performed better in off design flow conditions.
\end{abstract}

\section{Introduction}

In this paper, we report recent progresses and improvements made by applying state estimation and proper orthogonal decomposition (POD) in the development and experimental validation of a reduced-order model based control of the resonant subsonic flow over a shallow cavity. This work continues and expands our previous ones based on numerical data (Yuan et al. 2005) and experimental data (Caraballo et al. 2005,) towards the design and implementation of a feedback controller by applying system identification and modeling techniques to direct measurements of physical flow quantities. This work is part of a larger multidisciplinary effort in the development of a basic understanding and implementation of feedback flow control techniques (Samimy et al. 2004).

Successful application of feedback control is widespread in areas such as robotics, aerospace, telecommunication, transportation systems, manufacturing systems, and chemical processes. Recently, various

\footnotetext{
* Grad. Student, Dept. of Mechanical Eng., Student Member AIAA

Grad. Student, Dept. of Electrical and Computer Eng.

* Grad. Student, Dept. of Mechanical Eng., Student Member AIAA

$\S$ Post Doctoral Researcher, Dept. of Mechanical Eng., Member AIAA

** Assistant Professor, Dept. of Electrical and Computer Eng., Member AIAA

† Senior Aerospace Engineer, AFRL/VACA, Senior Member AIAA

$\$$ Professor, Dept. of Mechanical Eng., Director of GDTL, Associate Fellow AIAA, Corresponding author: samimy.1@osu.edu
} 
attempts have been made to apply feedback control techniques to aerodynamic flow phenomena (Gad-El-Hak 2000, Cattafesta et al. 2003). In contrast with open-loop flow control, which can produce useful results but lacks the responsiveness or the flexibility needed for application in dynamic flight environments, closed-loop flow control appears to be suited for the successful management of flow in many applications due to its adaptability to variable conditions and to its potential for significantly reducing the power required for controlling the flow (Cattafesta et al. 1997). However, the tools of classical control systems theory are not directly applicable to fluid flow systems which display spatial continuity and nonlinear behavior, and pose formidable modeling challenges due to their infinite dimensionality, the complexity introduced by the Navier-Stokes equations, and the peculiar characteristics of the measurement and actuation devices. In order to design and successfully implement a closed-loop control strategy, it is necessary to obtain simple dynamical models of the system, which on one hand capture the important dynamic characteristics of the flow and the effect of the actuation, and on the other hand are simple enough to be used for model-based feedback control.

The benchmark case selected in our study is the flow over a shallow cavity (Samimy et al. 2004), a configuration present in many practical applications that benefits from having been extensively studied by several researchers (e.g. Rossiter 1964, Heller and Bliss 1975, Cattafesta et al. 2003). This flow is characterized by a strong coupling between flow dynamics and flow-generated acoustic field that produces a self-sustained resonance known to cause, among other problems, store damage and airframe structural fatigue in weapons bays. A comprehensive review of this phenomenon and of different control and actuation strategies developed for its suppression is given in Cattafesta et al. (2003).

The approach we follow in the development of a reduced order model for the cavity flow is based on proper orthogonal decomposition (POD). This technique extracts information on the coherent structures, which are the most dominant characteristic of the flow and the only entities that can effectively be controlled, on the basis of the spatial correlation tensor of the velocity field in the flow. It represents the dynamics of flow field as a set of spatial eigenmodes that are modulated by time coefficients obtained by projecting the instantaneous flow fields onto the POD basis. Obtaining a time varying description would require simultaneous, real-time flow measurements at every spatial point. While this is feasible using data from numerical simulations, it becomes an extremely challenging task with data obtained experimentally even in low-speed flows (Thurow et al. 2005). In this case the obstacle can be surmounted by exploiting stochastic estimation methods that correlate the velocity field with a variable that can be measured continuously in time, such as surface pressure (Glauser et al. 2004, Ukeiley and Murray 2005, Caraballo et al. 2005).

The state equation employed for controller design is in the form of a system of nonlinear ordinary differential equations for the time coefficients. It is obtained by using the Galerkin projection method to project the governing flow equations with velocities approximated by the POD description onto the spatial basis. The system of equations can be recast in a form expressing the control input explicitly as required to apply the tools of control theory for the development of feedback control.

The procedure that we are using is summarized in more details in Caraballo et al. (2005). The state equation (i.e. the POD time coefficients) is estimated in real-time from dynamic surface pressure measurements by employing the stochastic estimation technique with correlations obtained from off-line simultaneous particle image velocimetry (PIV) and dynamic pressure measurements at several locations on the surface of the cavity.

Equilibrium analysis led to the linearization of the reduced-order model around the equilibrium point. A simpler model for controller design was then obtained by shifting the origin of the coordinates to the equilibrium point. This corresponds to removing the effect of the mean flow from the low-order model, and considering the local behavior of the system around the mean flow. The availability of real-time estimates of the state of the model allowed the use of linear state feedback control. To this aim we designed and tested experimentally a linear-quadratic optimal state feedback controller.

From the results obtained we can conclude that the controller significantly reduces the resonance peak of the Mach 0.3 single Rossiter mode, for which it was designed, by switching it to a multi-mode resonance. The controller seems also to be quite robust, as it can control the flow with some variations in the flow Mach number. In this paper we report further improvements to the technique that we have developed and used. Among these are a better convergence of the time coefficients calculated from the Galerkin system, enhanced control performance and a better scaling of the control gain which is closer to the unity theoretical value.

In the next sections we will introduce the flow facility used in this study and then focus on the POD and Galerkin methods adopted for deriving the reduced-order model, and the stochastic estimation approach used for real-time estimate of the model variables directly from dynamic pressure measurements. This is followed by the design and application of the linear-quadratic controller and by the presentation and discussion of the results. 


\section{The experimental facility}

The experimental facility is described in detail in Debiasi and Samimy (2004). It is an instrumented, optically accessible wind-tunnel that operates in a blow-down fashion with atmospheric exhaust. The filtered, dried air is conditioned in a stagnation chamber before entering a smoothly contoured converging nozzle to the 2 inch by 2 inch test section. The facility can run continuously in the subsonic range between Mach 0.20 and 0.70 and transonic and supersonic applications are possible by changing modular components.

A shallow cavity is recessed in the test section with a depth $D=12.7 \mathrm{~mm}$ and length $L=50.8 \mathrm{~mm}$ for a length to depth aspect ratio $L / D=4$. For control the cavity shear-layer receptivity region is forced by a 2-D synthetic-jet type actuator issuing at 30 degrees relative to the main flow from a $1 \mathrm{~mm}$ slot embedded in the cavity leading edge and spanning the width of the cavity, Fig. 1. A Selenium D3300Ti compression driver provides the mechanical oscillations necessary to create the zero net mass, non-zero net momentum flow for actuation. The actuator signals are produced by either a BK Precision 3011A function generator for open-loop forcing or by a dSPACE 1103 DSP control board in closed-loop studies and are amplified by a Crown D-150A amplifier.

The "snapshots" of the flow field, required for the development of the low dimensional model, are acquired and processed using a LaVision Inc. PIV system. Details of the PIV system, procedure, and results are presented in Little et al. (2006). The main flow is seeded with Di-Ethyl-Hexyl-Sebacat (DEHS) particles by using a 4-jet atomizer upstream of the stagnation chamber. This location allows homogenous dispersion of the submicron particles throughout the test section. A dual-head Spectra Physics PIV-400 Nd:YAG laser operating at the $2^{\text {nd }}$ harmonic $(532 \mathrm{~nm})$ is used in conjunction

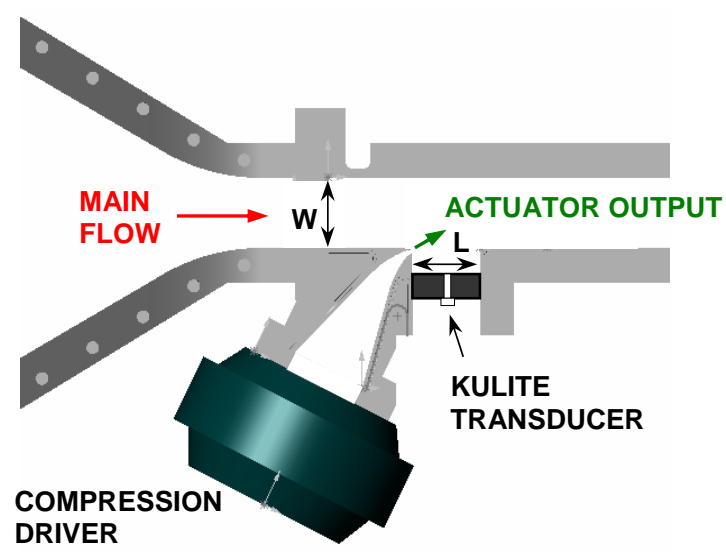

Figure 1. Scaled drawing of the experimental set up showing the incoming flow, the actuation location (at the receptivity location of the free shear layer formed over the cavity), and other geometrical details. with spherical and cylindrical lenses to form a thin $(\sim 1 \mathrm{~mm})$, vertical sheet spanning the streamwise direction of the cavity at the middle of test section width. In order to minimize beam reflections, a small slot cut into the cavity floor allows the laser sheet to exhaust and diffuse in a sealed light-trap. The time separation between the lasers pulses used for PIV can be tuned according to the flow velocity. For Mach 0.30 flow this value is 1.8 microseconds. Two images corresponding to the pulses from each laser head were acquired by a 2000 by 2000 pixel CCD camera equipped with a $90 \mathrm{~mm}$ macro lens with a narrow band-pass optical filter. The images were divided into 32 by 32 pixel interrogation windows which contained 6-10 seed particles each. For each image sub regions were cross correlated by using multi-pass processing with $50 \%$ overlap. The resulting vector fields were post-processed to remove any remaining spurious vectors. This setup gave a velocity vector grid of 128 by 128 over the measurement domain of $50.8 \mathrm{~mm}$ which translates to each velocity vector being separated by approximately $0.4 \mathrm{~mm}$.

Flush-mounted Kulite transducers were placed on various locations on the walls of the test section for dynamic pressure measurements. Figure 2 shows the locations of the transducers used in this study. All these transducers have an almost flat frequency response up to about $50 \mathrm{kHz}$ and are powered by a dedicated signal conditioner that amplifies and low-pass filters at $10 \mathrm{kHz}$ their signals.

For state estimation, dynamic pressure measurements were recorded simultaneously with the PIV measurements using a National Instruments (NI) PCI-6143 S-Series data acquisition board mounted on a Dell Precision Workstation 650. The system allows synchronous sampling of 8 channels with a maximum sampling frequency of 250 $\mathrm{kHz}$ per channel. Each pressure recording was band-pass filtered between $100 \mathrm{~Hz}$ and $10 \mathrm{kHz}$ to remove spurious frequency components. In the current study 1000 PIV snapshots were recorded for each flow/actuation condition explored. For each PIV snapshot 128 pressure samples from the laser Q-switch signal and from each of the

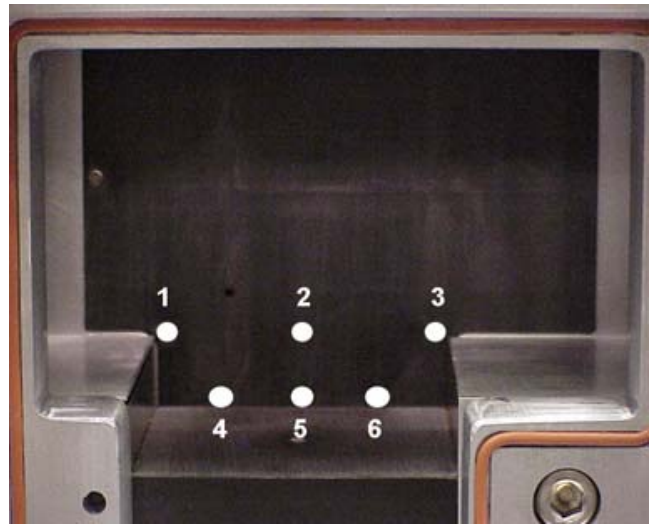

Figure 2. Location and numbering of Kulite pressure transducers in the cavity flow. 
transducers of Fig. 2 were acquired at $50 \mathrm{kHz}$. The NI board was triggered by a programmable timing unit (PTU) housed in the PIV system that activated the beginning of the acquisition to allow the Q-switch TTL to fall approximately in the middle of the 128 data points. The simultaneous sampling of the laser Q-switch signal with the pressure signals allows for each snapshot the identification of the section of pressure time traces corresponding to the instantaneous PIV velocity field. Additional, longer recordings of 262,144 samples per channel acquired at 200 $\mathrm{kHz}$ were also used to derive SPL spectra as described in Debiasi et al. (2004).

For closed-loop control of the flow a dSPACE 1103 DSP board connected to the Dell Precision Workstation 650 was used. This system utilizes four independent, 16-bit A/D converters each with 4 multiplexed input channels and allows simultaneous acquisition and control processing of 4 signals and almost simultaneous, due to multiplexing, acquisition and processing of additional signals at a rate up to $50 \mathrm{kHz}$ per channel to produce at the same rate a control signal from a 14-bit output channel. Similar to state estimation pressure data, the pressure signals were bandpass filtered between $100 \mathrm{~Hz}$ and $10 \mathrm{kHz}$ to remove spurious frequency components.

\section{Reduced-order Modeling}

Reduced-order models of the flow were derived from PIV and surface pressure measurements of the cavity flow as described by the authors in previous works (Samimy et al. 2004, and Caraballo et al. 2004, 2005). The approach is based on the combination of three separate tools. First, the POD method is used to obtain a spatial basis of the flow. Then, the POD expansion is combined with the Galerkin projection method to obtain the flow model which consists of a set of ordinary differential equations for the POD temporal coefficients. In this set of equations the control input appears explicitly to facilitate the design of the feedback control algorithm. Finally stochastic estimation is used to estimate these temporal coefficients based on real time surface pressure measurements.

\section{A. POD Method}

This is the first tool used to obtain the reduced-order model. In recent years POD has been used in the study of several turbulent flows and in the development of feedback control for them. The method was introduced by Lumley (1967) as an objective way to extract large-scale structures in a turbulent flow. The technique originally was developed for data sets of large sample time $\mathcal{T}$ at a few spatial locations $\mathcal{L}$. More details on the fundamentals of this method can be found in Holmes et al. (1996) and Delville et al. (1998). The POD approach used in this investigation is the snapshot method of Sirovich (1987), which is an alternative way of obtaining the POD modes more suitable for highly spatially resolved data sets $(\mathcal{L}>>\mathcal{T})$ that can be obtained using numerical simulations or advanced laserbased flow diagnostics. A detailed description of the application of the Sirovich POD method to the current study case is given in Samimy et al. (2004) and Caraballo et al. (2004).

The POD method aims to describe the temporal-spatial evolution contained in $\mathcal{T}$ realizations of the fluctuating components of a flow variable (e.g. the fluctuating component $u$ ' of the streamwise velocity $u$ ) as combination of $N$ $<\mathcal{T}$ spatial modes (or eigenfunctions) $\varphi_{\boldsymbol{i}}(\boldsymbol{x})$, i.e. a reduced basis of modes that capture the coherent structures, the dominant features, present in the flow:

$$
u^{\prime}(x, t) \cong \sum_{i=1}^{N} a_{i}(t) \varphi_{i}(x)
$$

The time coefficients $a_{i}(t)$ are functions of time only and capture the time evolution of the corresponding coherent structures. The number of modes, $N$, used depends on the nature of the problem and the purpose of the model. The time coefficients of a known instantaneous flow filed can be obtained from:

$$
a_{i}(t)=\int_{D} u^{\prime}(x, t) \varphi_{i}^{*}(x) d x
$$

where * denotes complex conjugate. Equation (3.2) requires that the instantaneous flow field be measured or numerically calculated simultaneously at every point in the flow domain of interest.

For each of the flow conditions explored in this work, 1000 PIV snapshots of the flow field were acquired as described in the previous section and used in the derivation of the modes and their time coefficients. The increased number of snapshot compared to that (500) used in Caraballo et al. (2005) allowed complete convergence of the average turbulent kinetic energy of the flow in the shear layer region. Figure 3 shows how the mean turbulent kinetic energy at different location on the shear layer converges when more than 700 images are used. Figure 4 shows the 
first two modes of the normal fluctuating velocity of the cavity flow using 500 and 1000 snapshots. It can be noticed how increasing the number of snapshots helps to obtain a smoother (cleaner) representation of the structures capture by the modes, as the small scale effects are captured by the higher modes added to the system. However, as it was noticed before (Caraballo et al. 2005), there is a small effect on the energy content of the modes, especially for the lower order modes.

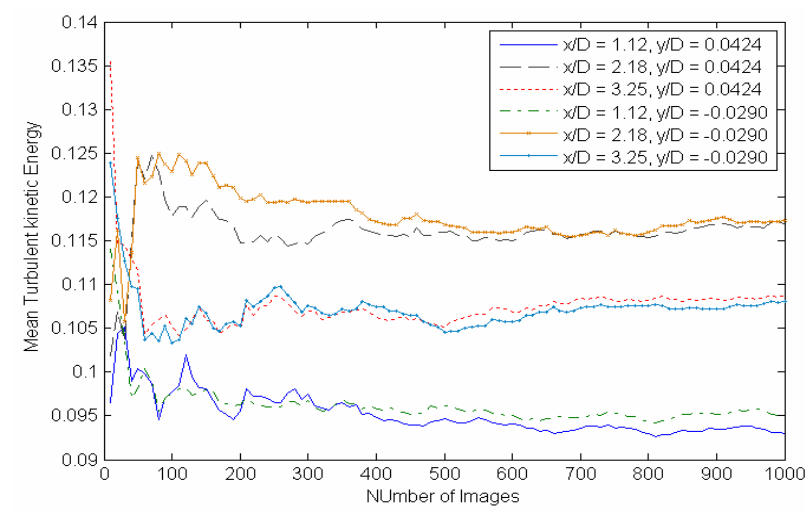

Figure 3. Mean turbulent kinetic energy of the cavity flow at different locations of the shear layer.
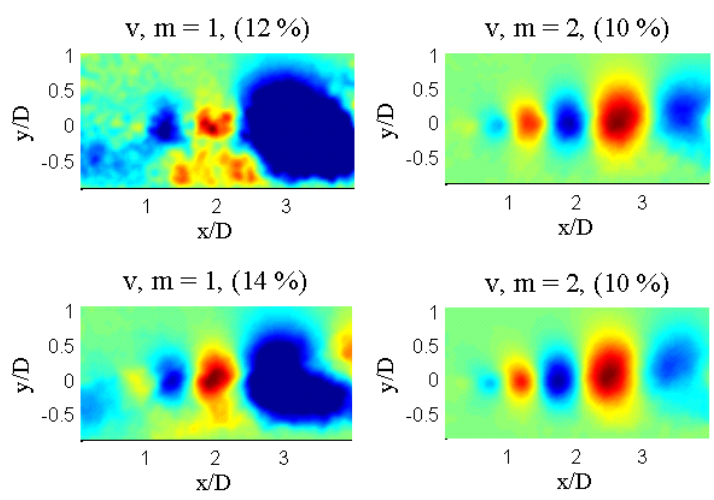

Figure 4. First two POD modes of the normal fluctuating velocity for the cavity flow: top 500 Snapshots; bottom 1000 snapshots.

\section{B. Galerkin Projection and Low Dimensional Model}

The Galerkin projection method was used to obtain a reduced-order model of the cavity flow dynamics, resulting in a system of ordinary differential equations for the time coefficients $a(t)=\left[a_{1}(t) a_{2}(t) \ldots a_{N}(t)\right]$. The method relies on the projection of the governing equations of the flow, the compressible Navier-Stokes equations in this case, onto the basis of POD modes. Detailed explanation on the derivation of this model is given in our earlier works (Samimy et al. 2004, and Caraballo et al. 2004). The form of the governing equations used here is based on the work of Rowley (2002), where the compressible Navier-Stokes equations are simplified and written as:

$$
\begin{aligned}
& \frac{D c}{D t}+\frac{\gamma-1}{2} c \nabla \bullet \boldsymbol{u}=0 \\
& \frac{D \boldsymbol{u}}{D t}+\frac{2}{\gamma-1} c \nabla c=\frac{\mu}{\rho} \nabla^{2} \boldsymbol{u}
\end{aligned}
$$

where $\boldsymbol{u}=(u, v)$ is the velocity vector and $c$ is the local speed of sound.

To apply the Galerkin method, first each flow variable is decomposed into its mean and fluctuating components, and then the POD expansion equation (3.1) is written for each of the fluctuating components. Next, the flow variables in (3.3) are replaced by the expanded expressions of mean and fluctuating components. The new form of the governing equations is then projected onto the basis of POD modes by taking the inner product of each term with the POD modes according to the vector norm (Rowley, 2002). This procedure yields a system of equations in which the control input is not separated from the rest of the flow, i.e. the control effect is implicit in the model. This is not useful for control law design. In order to derive a model where the control input appears explicitly in the equations, the spatial sub-domain where the control is active is separated from the rest of the field (Caraballo et al. 2005), yielding a system in the following form:

$$
\dot{a}(t)=F+G a(t)+\left[\begin{array}{c}
a^{T}(t) H^{1} a(t) \\
\vdots \\
a^{T}(t) H^{N} a(t)
\end{array}\right]+B \Gamma(t)+\left[\begin{array}{c}
\left(\bar{B}^{1} \Gamma(t)\right)^{T} a(t) \\
\vdots \\
\left(\bar{B}^{N} \Gamma(t)\right)^{T} a(t)
\end{array}\right],
$$

where the matrices of constant coefficients $F, G, H^{i}, B$ and $\bar{B}^{i}, i=1, \ldots N$, are obtained from the Galerkin projection, and $\Gamma(t)$ is the control input applied at the forcing location, see Yuan et al. (2005). Equation (3.4) 
represents a model of the cavity flow in terms of the time coefficients $a(t)$ obtained with POD from $\mathcal{T}$ time uncorrelated PIV data sets.

By using a finite number $N$ of modes to describe the flow one not only loses some flow details, but also fails to capture the energy transfer process between the $N$ retained modes and the neglected ones. Therefore, an additional viscous term, the modal eddy viscosity (Noack et al. 2004), was added to the model to maintain the overall flow energy balance and to compensate for other small errors introduced in the derivation of the model. This additional viscous term is added to the viscous term present in the flow equation, and is obtained by a modal energy balance as presented in Noack et al. 2004.

The system (3.4) was solved to check the evolution and convergence of the time coefficients and to compare it to the corresponding values obtained with (3.2) from the PIV snapshots. The time coefficients evolved to bounded values in most cases when 4 through 10 modes were used in (3.4). Figure 5 shows the evolution of the time coefficient of the first mode for the baseline Mach 0.3 flow when using $N=$ 4. After an initial transient this coefficients oscillates around close to zero with an amplitude comparable to the time coefficients obtained with (3.2) from the PIV snapshots. This is a better result than the one we previously obtained by using 500 snapshots of the same flow (Caraballo et al. 2005) which is also shown in Fig. 5 for the sake of comparison. However, we observed that the frequency of oscillation of the new set is slightly lower than the experimental value, which we relate to the additional viscous dissipation introduced into the model. It was

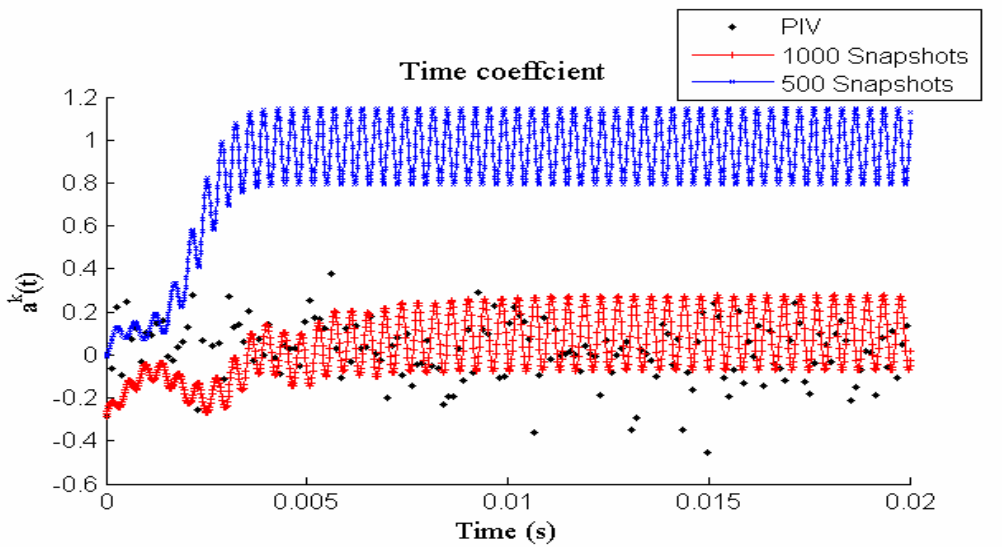

Figure 5. First time coefficient form GS. Black (dots) PIV, Red (+) 1000 Snapshots and Blue (x) 500 Snapshots.

observed that the system trajectories converged to the same behavior, irrespective of the initial condition of the time coefficient used for the solution of (3.4), showing the occurrence of a stable limit cycle. Similar results were obtained for the time coefficients of the other three modes of the baseline Mach $0.3 \mathrm{flow}$, and for the modes of the same flow with different types of forcing.

The overall effect that these improvements had in the controller design and implementation will become clear later. Finally, we elected to use the $N=4$ for the model used to design the controller so to simplify the control algorithm and its experimental implementation.

\section{Stochastic Estimation}

For the implementation of the feedback controller, availability in real-time of the time coefficient is required. This was achieved using Stochastic Estimation (SE). Stochastic estimation, proposed by Adrian (1979) as a method to extract coherent structures from a turbulent flow field, estimates flow variables at any location by using statistical information about the flow at a limited number $\mathcal{L}^{*}$ of locations. The method has been used to study various flows (e.g. Adrian and Moin 1988, Cole and Glauser 1998), and as a complementary tool for POD modeling to estimate the time coefficients from experimental measurements in subsonic jets (Picard and Delville, 2000) and in cavity flows (Samimy et al. 2004 and Ukeiley and Murray, 2005). In recent years, it has also been used to estimate the time coefficient of POD models for feedback flow control (Glauser et al. 2004, Siegel et al. 2005, and Caraballo et al. 2005).

In this study, quadratic SE was employed to estimate the time coefficients of the flow model (3.4) directly from real-time measurements of surface pressure fluctuations at a small number $\mathcal{L}^{*}$ of locations. The estimates of the time coefficients can be written in the following form:

$$
\hat{\boldsymbol{a}}_{\boldsymbol{i}}(\boldsymbol{t})=\boldsymbol{C}_{\boldsymbol{i} \mathbf{k}} \boldsymbol{p}_{\boldsymbol{k}}^{\prime}(\boldsymbol{t})+\boldsymbol{D}_{\boldsymbol{i k l} \mathbf{l}} \boldsymbol{p}_{\boldsymbol{k}}^{\prime}(\boldsymbol{t}) \boldsymbol{p}_{\boldsymbol{l}}^{\prime}(\boldsymbol{t}) \quad i=1 \ldots N, \quad k, l \ldots=1 \ldots \mathcal{L}^{*},
$$

where $C, D \ldots$ are the matrices of the estimation coefficients obtained by minimizing the average mean square error $e_{i}$ between the values of $a_{i}\left(t_{r}\right)$ obtained with (3.2) for the time of PIV image capture, and the estimated ones $\hat{a}_{i}\left(t_{r}\right)$ at the same times, that is, 


$$
e_{i}=\left\langle\left[\hat{a}_{i}\left(t_{r}\right)-a_{i}\left(t_{r}\right)\right]^{2}\right\rangle \quad r=1 \ldots \mathcal{T}
$$

To calculate the matrices in (3.5), only $\mathcal{L}^{*}$ pressure values taken simultaneously with of PIV measurements were used from each of the 1000 simultaneous PIV-pressure acquisitions. The procedure to obtain the estimation matrices is described in more detail in Caraballo et al. (2004). In our experimental setup, real-time measurements of the surface pressure were obtained at $\mathcal{L}^{*}=6$ locations of interest in the cavity test section shown in Fig. 2.

The stochastic estimation method has been previously tested for the cavity flow under investigation, showing good agreement in the estimation of the time coefficients (Caraballo et al. 2005). When the method was applied to the new data set the estimated coefficients of all four modes remained close the values obtained from the PIV images.

\section{Controller Design and Implementation}

In this section, we present a model-based controller design, and discuss real-time implementation results. The control design approach has been already presented in Yuan et al. (2005) and Caraballo et al. (2005) in details, and thus it will be only sketched briefly. The design procedure includes equilibrium computation, coordinates transformation, linear approximation and linear-quadratic state feedback control design. With respect to Yuan et al. (2005) and Caraballo et al. (2005), in this work the design procedure was implemented on a more accurate Galerkin model obtained using the larger experimental data set (PIV data). The real time implementation of the new controller showed a sizable improvement over the preliminary results presented in Caraballo et al. (2005). The nominal flow model used here for control design is the nonlinear state space model for the baseline flow at Mach 0.3 given by (3.4), with $N=4$.

\section{A. Equilibrium analysis and model simplification}

Performing equilibrium analysis and coordinate transformation on model (3.4), the constant term $F$ is removed from the model, shifting the origin of the coordinates to the equilibrium point. The resulting simplified state space model in the new set of coordinates $\tilde{a}=a-a_{0}$ reads as

$$
\dot{\tilde{a}}=\tilde{G} \tilde{a}+\left[\begin{array}{c}
\tilde{a}^{T} H^{1} \tilde{a} \\
\vdots \\
\tilde{a}^{T} H^{4} \tilde{a}
\end{array}\right]+\tilde{B} \Gamma+\left[\begin{array}{c}
\left(\bar{B}^{1} \Gamma\right)^{T} \tilde{a} \\
\vdots \\
\left(\bar{B}^{4} \Gamma\right)^{T} \tilde{a}
\end{array}\right],
$$

where $a_{0}$ is the equilibrium point computed for the model (3.4) and

$$
\tilde{G}=G+\left[\begin{array}{c}
a_{0}^{T}\left(H^{1}+\left(H^{1}\right)^{T}\right) \\
\vdots \\
a_{0}^{T}\left(H^{4}+\left(H^{4}\right)^{T}\right)
\end{array}\right], \quad \tilde{B}=B+\left[\begin{array}{c}
\left(\bar{B}^{1}\right)^{T} a_{0} \\
\vdots \\
\left(\bar{B}^{4}\right)^{T} a_{0}
\end{array}\right] .
$$

Clearly, the modified model has an equilibrium at the origin, which is more convenient for controller design and stability analysis. The reader is referred to Caraballo et al. (2005) for a detailed description of the model simplification techniques.

\section{B. Linear quadratic state feedback control}

A linear approximation of (4.1) at the origin is readily obtained as

$$
\dot{\tilde{a}}=\tilde{G} \tilde{a}+\tilde{B} \Gamma .
$$

The eigenvalues of the unforced system have been computed as 


$$
\lambda(\tilde{G})=\left[\begin{array}{c}
1596.6+7023.1 \mathrm{i} \\
1596.6-7023.1 \mathrm{i} \\
-3652 \\
-879.9
\end{array}\right] .
$$

The presence of two unstable conjugate eigenvalues implies, as expected, that the mean flow (corresponding to the equilibrium $a_{0}$ ) is an unstable solution for the Galerkin system (3.4).

Since the pair $(\tilde{G}, \tilde{B})$ is controllable, linear state-feedback design based on the linearized model (4.3) offers a simple approach to the design of a controller for the nonlinear model (4.1). It should be noted that the availability of estimates of the states of the Galerkin model (3.4) obtained via stochastic estimation, renders state-feedback control a feasible methodology for the problem at issue. Linear-quadratic (LQ) optimal control, in turn, offers a convenient and well-established methodology for obtaining the controller gains. The LQ design computes the gain matrix $K$ such that the state-feedback law

$$
\Gamma(t)=-K \tilde{a}(t)
$$

minimizes the quadratic cost function

$$
J_{c}(\tilde{a}, \Gamma)=\int_{0}^{\infty}\left(\tilde{a}^{T} W_{\tilde{a}} \tilde{a}+W_{\Gamma} \Gamma^{2}\right) d t,
$$

where $W_{\tilde{a}}>0$ and $W_{\Gamma}>0$ are positive definite weighting functions for the state vector and the control signal, respectively. Minimization of $J_{c}$ results in asymptotic stabilization of the origin, while keeping the control energy small. In our design, the weights have been chosen as $W_{\tilde{a}}=I_{4 \times 4}$ and $W_{\Gamma}=1$, and the corresponding control gain reads as

$$
K=\left[\begin{array}{llll}
-56.2176 & 8.8345 & -417.2384 & -12.7746
\end{array}\right] .
$$

Applying the state feedback control (4.5) to the linearized system (4.3) results in mirroring all the right-half plane eigenvalues of the matrix $\tilde{G}$ to the left half plane, as indicated in Fig. 6. Figure 7 shows the simulation results obtained by applying the state feedback control (4.5) to the finite-dimensional nonlinear model (3.4), which indicate that the closed-loop state trajectories $a(t)$ converge to the equilibrium point

$$
a_{0}=\left[\begin{array}{llll}
-0.5036 & 0.2788 & -0.1930 & 0.4980
\end{array}\right]^{T} .
$$

It can be concluded that, in principle, the LQ controller (4.5) designed for the linear approximation (4.3) succeeds in stabilizing the equilibrium of the four-modes nonlinear Galerkin system (3.4).

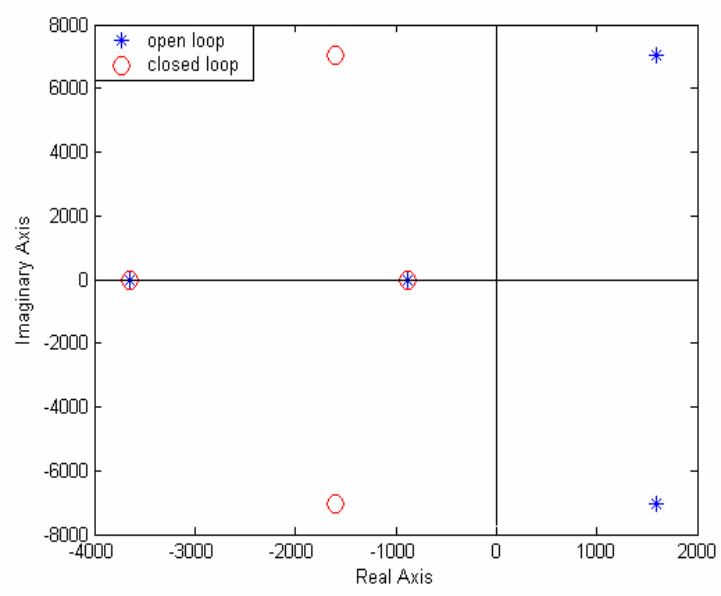

Figure 6. Eigenvalues of the open loop system and closed loop system.

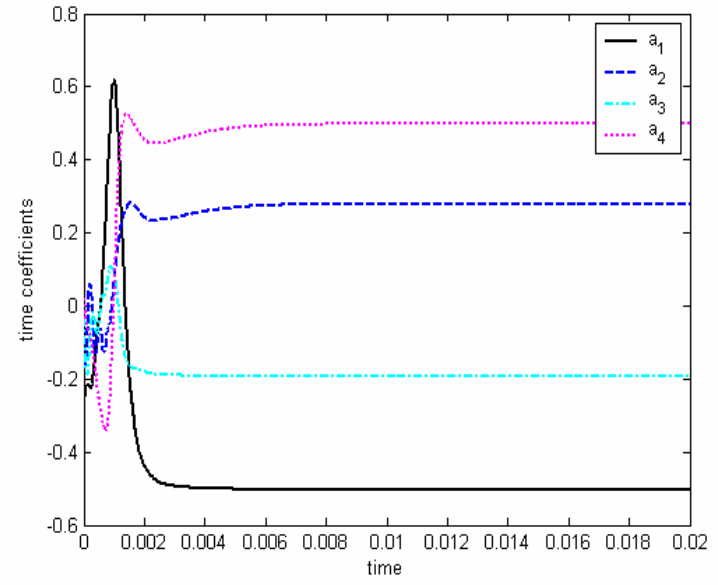

Figure 7. Time coefficient solutions of the closed loop simulation results. 


\section{Real time control results}

Before presenting the experimental results, it is worth summarizing the structure of the model-based controller thus derived. As depicted in Fig. 8, the model-based controller includes a stochastic estimation subsystem and a feedback from the estimated states. The estimate $\hat{\tilde{a}}$ of the deviation from the equilibrium of the time coefficients of the Galerkin model, required to implement the feedback law (4.5), may be in principle obtained by means of stochastic estimation by first estimating $\hat{\boldsymbol{a}}(\boldsymbol{t})$ from raw pressure measurements using equation (3.5), and then subtracting the equilibrium value $a_{0}$ computed from the model data. However, in implementing the controller, it was found that subtracting the equilibrium values from the estimated ones is not required since the values

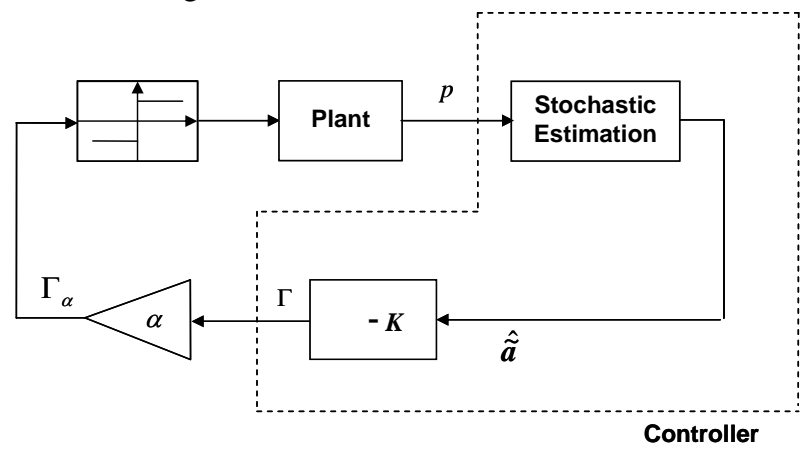

Figure 8. Diagram of the closed loop system with LQ state feedback control. estimated with equation (3.5) from fluctuating pressure measurements (that neglect the effect of mean flow) are already oscillating around zero for each mode. That is, equation (3.5) naturally produces the values of $\hat{\tilde{a}}$ form the experimental measurements. To protect the acoustic actuator, a saturation function is employed in cascade with the controller output. To prevent saturating the control input at all time, the controller is detuned using a constant scaling factor $\alpha$, similarly to what has been presented in Caraballo et al. (2005). The largest possible scaling factor in this study has been found to be $\alpha=0.265$, and the corresponding scaled control is in the form

$$
\Gamma_{\alpha}(t)=-\alpha K \hat{\tilde{a}}(t) .
$$

The performance of the scaled control law (4.9) has been tested in closed-loop experiments for different flows in the neighborhood of Mach 0.3, the nominal case for which the controller was designed. Figure 9 compares examples of SPL reduction obtained by the LQ state feedback control (left column) with the open-loop optimal forcing control for the same flow conditions presented in Debiasi et al. 2004 (right column). The thin red line yields the SPL of the unforced baseline flow recorded at the center of the cavity (location 5 in Fig. 2), whereas the thick line corresponds to the SPL of the flow at the same location under state feedback control (blue) or optimal forcing control (green). The baseline flows at Mach 0.3 (a), Mach 0.27 (b), and Mach 0.32 (c) exhibit a strong, single-mode resonant peak. The frequency of this peak slightly increases with flow velocity from about 2700 to about $2900 \mathrm{~Hz}$ as predicted by the Rossiter formula. For the nominal case at Mach 0.3 (a), LQ control reduces the resonant peak by more than 15 $\mathrm{dB}$, accompanied by the emergence of two additional peaks with frequencies around $2100 \mathrm{~Hz}$ and $3100 \mathrm{~Hz}$, with lower SPL level than the original frequency. The same general characteristics and benefits are maintained when the control is applied to the lower Mach 0.27 flow (b) or to the higher Mach 0.32 one (c). Controlling the same flows with open-loop optimal forcing (right column) produces less noticeable reductions of the resonant peaks at Mach 0.30 and 0.27 and always introduces peaks at the forcing frequency higher than the highest spectral peaks with LQ control.

The scaled LQ state feedback control has been shown to successfully reduce the dominant Rossiter peak and have good robustness under different flow conditions, as shown by Fig. 9. The impact of the scaling factor $\alpha$ from the control systems point of view has been analyzed in our previous paper (Caraballo et al. (2005)). An improvement of the scaling factor from 0.05 to 0.265 has been achieved in this study, resulting in an increased attenuation of the resonant peak observed in the experiments. The analysis reveals that the scaled LQ control with $\alpha$ $=0.265$ is still not a stabilizing controller, although it has moved the eigenvalues of the linearized closed-loop system (4.3)-(4.9) closer to the imaginary axis (compare Figure 10 to the results given in Caraballo et al. (2005)). Meanwhile, the scaled control with $\alpha=0.265$ reduced the amplitude of the oscillations in the nonlinear closed-loop model (4.1)-(4.9), to a great extent, as shown in Fig. 11. It is evident that, though the scaled LQ control is not able to asymptotically stabilize the origin of the nonlinear model (4.1), it nevertheless provides a significant reduction of the amplitude of the stable limit cycle. The experimental results agree with this interpretation, by showing a reduction of the amplitude of the Rossiter peak, Fig. 9. 

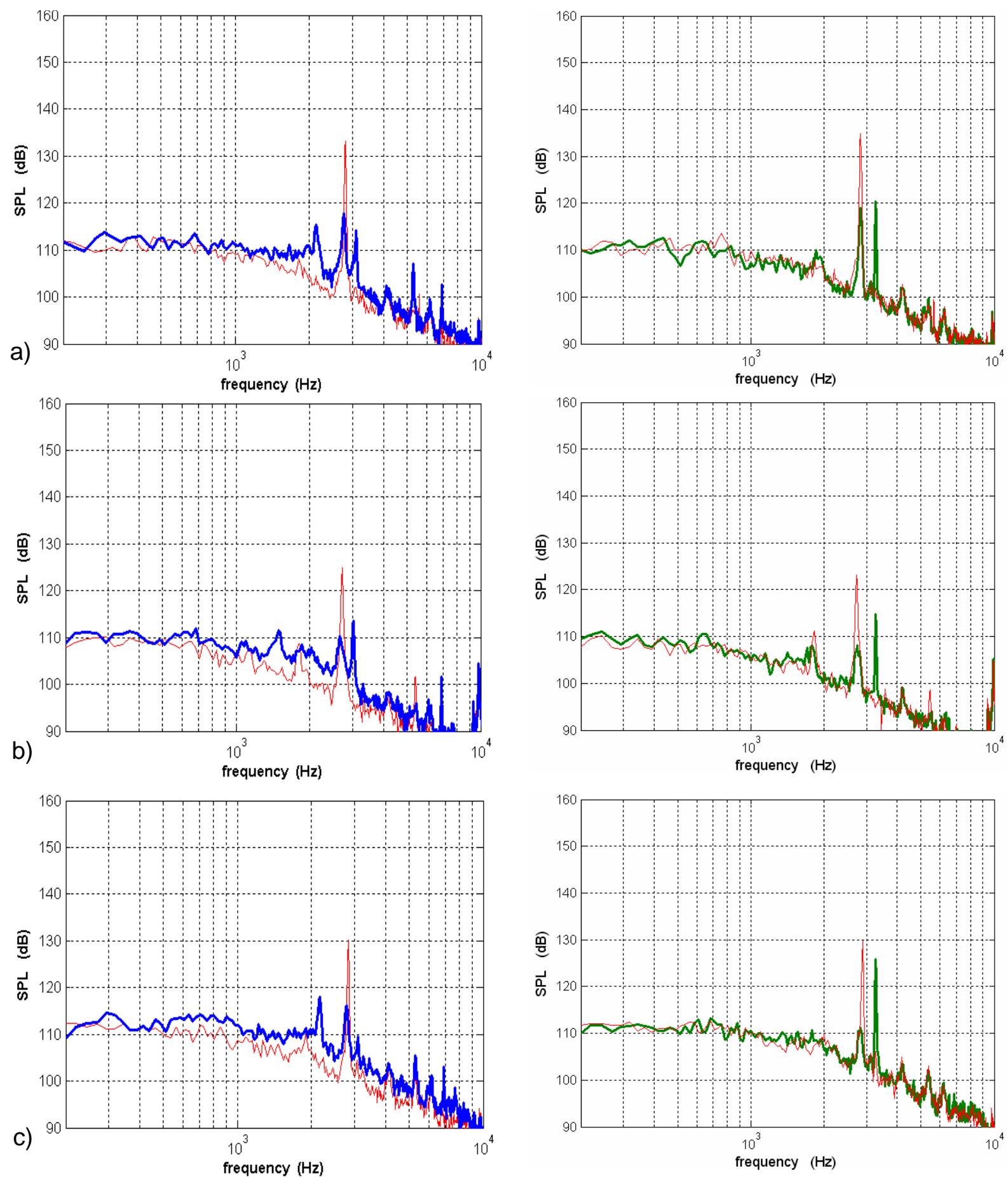

Figure 9. Effect of LQ control with 4 modes (left plots) and of optimal open loop forcing (right) on cavity flow with different Mach number; thin (red) line is the unforced flow SPL spectrum and thick (blue or green) line is the spectrum with control at: a) Mach 0.30 (design condition); b) Mach 0.27; c) Mach 0.32. 


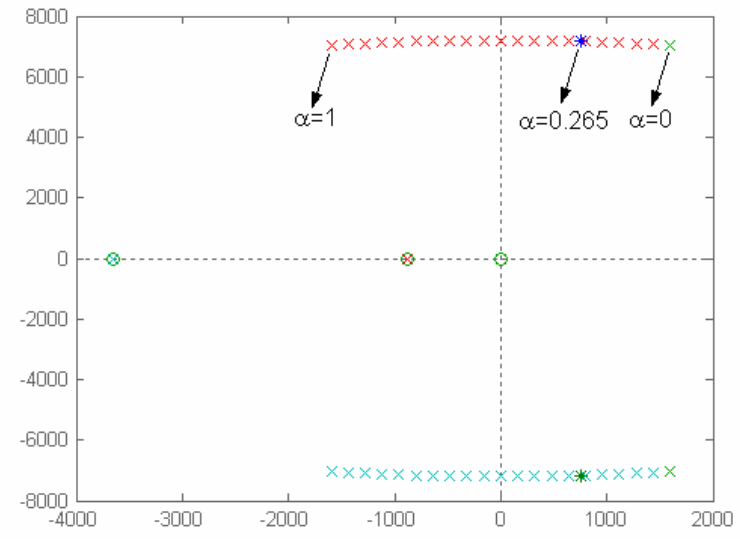

Figure 10. Eigenvalues of the closed loop systems with different scaling factor $\alpha$.

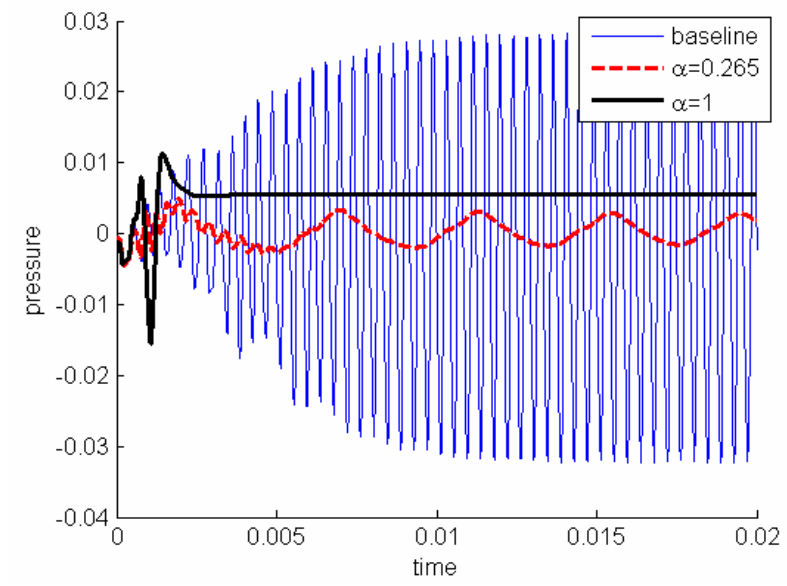

Figure 11. Closed loop responses at $P_{3}$ with different scaling factor $\alpha$.

\section{Conclusions}

The paper presents the most recent progress made by the flow control group at the Collaborative Center of Control Science at The Ohio State University in the development of reduced-order model based feedback flow control. A larger set of data composed of 1000 simultaneous PIV - pressure measurements were used to develop the feedback controller based on the reduced order model. The reduced-order model was obtained using the same methodology presented in our previous work, and is based on the use of snapshot based Proper Orthogonal Decomposition (POD) of PIV data in conjunction with Galerkin projection of the Navier-Stokes equations onto the POD eigenfunctions. The stochastic estimation method was used for real-time estimate of the time coefficients of the model from dynamic surface pressure measurements. It was observed that increasing the number of snapshots used for the derivation of the reduced order model improved the convergence of the model for the estimation of the time coefficients. The time coefficients for the model are oscillating around zero instead of about 1 as they did before. This suggests that the model is better predicting the experimental behavior of the flow. Equilibrium analysis led to the linearization of the reduced-order model around the equilibrium point. A simpler model for controller design was obtained by shifting the origin of the coordinates to the equilibrium point. This corresponds to removing the effect of the mean flow from the low-order model, and considering the local behavior of the system around the mean flow. The availability of real-time estimates of the state of the model allowed the use of linear state feedback control. To this aim we designed and tested experimentally a linear-quadratic optimal state feedback controller. From the results obtained we can conclude that the controller significantly reduces the resonance peak of the Mach 0.3 single-mode, for which it was designed, while introducing a couple of lower amplitude peak at $2100 \mathrm{~Hz}$ and $3100 \mathrm{~Hz}$. As before, the controller seems to be quite robust, as it can control the flow with some variations in the flow Mach number. It was also noted that augmenting the number of snapshots used in the process increases the value of the scaling factor $\alpha$ for the real time implementation to values closer to unity suggesting that that the model is a better representation of the real flow.

The improvements achieved so far indicate that a richer model and a better estimation of the states of the Galerkin model have been used in this study, in comparison with our earlier work. While the current results are quite encouraging, they could be further improved: at the present, models based on snapshots from several forced flow cases and combination of flow cases are being developed and tested. The results obtained so far are promising, but further analysis, interpretation, and development are required. Additional methods for the control separation and multi-time estimation of the time coefficient are being pursued as well.

\section{Acknowledgments}

This work is supported in part by the AFRL/VA and AFOSR through the Collaborative Center of Control Science (Contract F33615-01-2-3154).

The authors would like to thank Drs. James DeBonis and R. C. Camphouse, and John Casey and Cosku Kasnakoglu for help and fruitful discussions. 


\section{References}

1. Adrian, R. J., "On the Role of Conditional Averages in Turbulent Theory," Turbulence in Liquids, Science Press, Princeton, 1979.

2. Adrian, R. J., and Moin, P., "Stochastic estimation of organized turbulent structure: Homogeneous shear Flow," Journal of Fluid Mechanics, Vol. 190, 1988, pp. 531-559.

3. Caraballo, E., Malone, J., Samimy, M., and DeBonis, J., “A Study of Subsonic Cavity Flows - Low Dimensional Modeling," AIAA Paper 2004-2124, June 2004.

4. Caraballo, E., Yuan, X., Little, J., Debiasi, M., Yan, P. Serrani, A., Myatt, J., and Samimy, M., "Feedback Control of Cavity Flow Using Experimental Based Reduced Order Model," AIAA Paper 2005-5269, June 2005.

5. Cattafesta III, L.N., Garg, S., Choudhari, M., and Li, F., "Active Control of Flow-Induced Cavity Resonance”, AIAA Paper 97-1804, June 1997.

6. Cattafesta III, L. N., Williams, D. R., Rowley, C. W., and Alvi, F. S., "Review of Active Control of Flow-Induced Cavity Resonance," AIAA Paper 2003-3567, June 2003.

7. Cole, D. R., and Glauser, M. N, "Application of Stochastic Estimation in the axisymmetric Sudden Expansion," Physics of Fluids, Vol. 10, No. 11, 1998, pp. 2941-2949.

8. Debiasi, M. and Samimy, M., "Logic-Based Active Control of Subsonic Cavity Flow Resonance," AIAA Journal, Vol. 42, No. 9, pp. 1901-1909, September 2004.

9. Debiasi, M., Little, J., Malone, J., Samimy, M., Yan, P., and Özbay, H., “An Experimental Study of Subsonic Cavity Flow Physical Understanding and Control," AIAA Paper 2004-2123, June 2004.

10. Delville, J., Cordier, L. and Bonnet, J.P., "Large-Scale-Structure Identification and Control in Turbulent Shear Flows," In Flow Control: Fundamentals and Practice, edited by Gad-el-Hak, M., Pollard A. and Bonnet, J., Springer-Verlag, 1998, pp. 199-273.

11. Gad-el-Hak, M., Flow Control - Passive, Active, and Reactive Flow Management, Cambridge University Press, New York, NY, 2000.

12. Glauser, M. N., Higuchi, H., Ausseur, J., and Pinier, J., "Feedback Control of Separated Flows (Invited)," AIAA Paper 2004-2521, June 2004.

13. Heller, H. H., and Bliss, D. B., "The Physical Mechanisms of Flow-Induced Pressure Fluctuations in Cavities and Concepts for their Suppression,” AIAA Paper 75-491, March 1975.

14. Holmes, P., Lumley, J.L., and Berkooz, G., "Turbulence, Coherent Structures, Dynamical System, and Symmetry," Cambridge University Press, Cambridge, 1996.

15. Little, J., Debiasi, M., and Samimy, M., "Flow Structure in Controlled and Baseline Subsonic Cavity Flows," AIAA Paper 2006-0480, January 2006.

16. Lumley, J. "The Structure of Inhomogeneous Turbulent Flows", Atmospheric Turbulence and wave propagation. Nauca, Moscow. 1967 166-176.

17. Noack, B., Tadmor, G., and Morzynski, M. ., "Low-Dimensional Models for Feedback Flow Control. Part I: Empirical Galerkin Models," AIAA Paper 2004-2408, June 2004.

18. Picard, C., and Delville, J., "Pressure Velocity Coupling in a Subsonic Round Jet;" International Journal of Heat and Fluid Flow, Vol. 21, pp. 359-364, 2000.

19. Rossiter, J.E., "Wind Tunnel Experiments on the Flow Over Rectangular Cavities at Subsonic and Transonic Speeds", RAE Tech. Rep. 64037, 1964 and Aeronautical Research Council Reports and Memoranda No. 3438, Oct. 1964.

20. Rowley, C. W., "Modeling, Simulation and Control of Cavity flow Oscillations", Ph.D. thesis, California Institute of Technology. 2002.

21. Samimy, M., Debiasi, M., Caraballo, E., Malone, J., Little, J., Özbay, H., Efe, M. Ö., Yan, P., Yuan, X., DeBonis, J., Myatt, J. H., and Camphouse, R. C., "Exploring Strategies for Closed-Loop Cavity Flow Control," AIAA Paper 2004-0576, January 2004.

22. Siegel, S., Cohen, K., Seidel, J., and McLaughlin, T., “Tow Dimensional Simulations of a Feedback Controlled D-Cylinder Wake," AIAA Paper 2005-5019, June 2005.

23. Sirovich, L. "Turbulence and the Dynamics of Coherent Structures", Quarterly of Applied Math. Vol. XLV, N. 3, 1987, pp. 561-590.

24. Thurow, B. S., Jiang, N., Lempert, W. R., and Samimy, M., "Development of Megahertz-Rate Planar Doppler Velocimetry for High-Speed Flows," AIAA Journal, Vol. 43 N³, March 2005, pp. 500-511.

25. Ukeiley, L., and Murray, N., "Velocity and Surface Pressure Measurements in an Open Cavity," Exp. In Fluids, Vol. 38, 2005, pp. 656-671.

26. Yuan, X., Caraballo, E., Yan, P., Özbay, H., Serrani, A., DeBonis, J., Myatt, J. H. and Samimy, M., "Reduced-Order Model-Based Feedback Controller Design for Subsonic Cavity Flows”, AIAA Paper 2005-0293, January 2005. 